\title{
Validação de método analítico por ELL-CG-EM para detecção de trialometanos decorrentes da cloração de águas contendo Microcystis
}

\author{
Analytical method and trihalomethanes formation by Microcystis \\ Elton Santos Franco'* ${ }^{10}$, Ana Flávia de Assis Ferreira' ${ }^{\circledR}$, Diego Francis Silva' ${ }^{\circledR}$, \\ Júlia Araújo Camargo' ${ }^{\circledR}$, Valter Lúcio de Pádua ${ }^{2}{ }^{(0}$, Jairo Lisboa Rodrigues ${ }^{1}{ }^{\circledR}$, \\ Mariandry del Valle Rodríguez ${ }^{1}{ }^{\oplus}$, Alessandra Giani $^{2}{ }^{\circledR}$
}

\section{RESUMO}

Estudos demonstram que a utilização do cloro em estações de tratamento de água (ETA) pode contribuir para a formação de subprodutos orgânicos halogenados indesejados, tais como os trialometanos (TAM), quando há presença dematéria orgânicaalgogênica, composta dealgase cianobactérias. A Microcystis aeruginosa é uma espécie de cianobactéria com frequentes registros em eventos de florações no país e é relacionada com a formação de TAM durante a cloração da água. Desse modo, este estudo teve como objetivo o desenvolvimento e a validação do método analítico por extração líquido-líquido para detecção e quantificação de TAM por cromatografia gasosa acoplada à espectrometria de massas (ELL-CG-EM), bem como a aplicação deste para avaliar a formação de TAM em ensaios de cloração de células de Microcystis aeruginosa, simulando situações em ETA. O método obteve baixo tempo de análise ( 12 minutos), excelente seletividade, precisão, repetitividade e sensibilidade, com possibilidade de aplicação para análises de rotina em detrimento de outras técnicas consideradas mais automatizadas. Foram observadas alta demanda de cloro durante os ensaios e elevada concentração dos subprodutos quando submetida à dose de cloro gasoso $\left(\mathrm{Cl}_{2}\right)$ de 2,5 e 5 mg. L', com destaque para o triclorometano, sendo outras espécies de monitoramento obrigatório não detectadas ou não formadas, o que pode ser justificado pela ausência de bromo.

Palavras-chave: estação de tratamento de água; demanda de cloro; cianobactérias; trialometanos; cromatografia gasosa acoplada à espectrometria de massas.

\begin{abstract}
Studies have shown that the use of chlorine in water treatment plants (WTPs) can contribute to formation of undesirable halogenated organic by-products, such as trihalomethanes (THMs), when algae and cyanobacteria are present. Microcystis aeruginosa is a cyanobacteria specie with frequent recordings of flowering events in the country and is related to the formation of THMs during water chlorination. Thus, the objective of this study was to develop and validate Liquid-Liquid Extraction the analytical method for detection and quantification of THMs by gas chromatography coupled to mass spectrometry (LLE-GC-MS), as well as the application of the method to evaluate the formation of TAMs from Microcystis aeruginosa cells chlorination tests, simulating situations in WTPs. The method obtained low time of analysis ( $<12$ minutes), excellent selectivity, precision, repeatability and sensitivity, with possibility of application for routine analysis to the detriment of other techniques considered more automated. High chlorine demand was observed during the tests and high concentration of by-products when submitted to the chlorine gas $\left(\mathrm{Cl}_{2}\right)$ dose of 2.5 and $5 \mathrm{mg} \cdot \mathrm{L}^{-1}$, with emphasis on trichloromethane. Other species of mandatory monitoring were not detected or not formed, justified by the absence of bromine.
\end{abstract}

Keywords: water treatment plants; chlorine demand; cyanobacterias; trihalomethanes; gas chromatography coupled to mass spectrometry.

\section{INTRODUÇÃO}

As atividades antrópicas têm afetado diretamente os corpos d'água, uma vez que os despejos de poluentes oriundos do aumento da produção de efluentes domésticos, agrícolas e industriais com alto teor de matéria orgânica e de nutrientes têm causado a proliferação excessiva de algas e cianobactérias em reservatórios e corpos hídricos usados para o abastecimento público. O principal agente oxidante utilizado nas estações de tratamento de água (ETA) para a inativação de microrganismos é o cloro. Isso se dá em virtude de seu baixo custo, praticidade de aplicação, residual persistente e efetividade quando comparado aos demais oxidantes (LIBÂNIO, 2010). 
Apesar dos benefícios no processo de controle de microrganismos e patógenos presentes na água usada com fins de abastecimento, estudos demonstram que a utilização do cloro pode contribuir para a formação de subprodutos orgânicos halogenados (SOH) indesejados, tais como os trialometanos (TAM), quando há presença de matéria orgânica natural (MON) na água. A MON pode ser derivada da decomposição das vegetações terrestre e aquática e da matéria orgânica algogênica (MOA), composta de algas e cianobactérias. Além das substâncias orgânicas às quais, tradicionalmente, atribui-se sua formação, estudos destacam e relacionam espécies de cianobactérias como parte importante na composição da MOA e também na formação de TAM e outros SOH (ZAMYADI et al., 2013; CORAL et al., 2013).

Os TAM constituem a principal classe de subprodutos em água tratada e uma das primeiras a serem descobertas. Os limites em águas para fins de abastecimento público variam em cada país: União Europeia (100 $\left.\mu \mathrm{g} . \mathrm{L}^{-1}\right)$, França $\left(100 \mu \mathrm{g} . \mathrm{L}^{-1}\right)$, Estados Unidos $\left(80 \mu \mathrm{g} . \mathrm{L}^{-1}\right)$, Brasil $\left(100 \mu \mathrm{g} . \mathrm{L}^{-1}\right)$

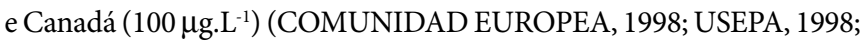
BRASIL, 2017a; HEALTH CANADA, 2017), sendo o triclorometano (TCM), o bromodiclorometano (BDCM), o dibromoclorometano (DBCM) e o tribromometano (TBM) os de monitoramento exigido nos mencionados padrões de potabilidade. Os TAM são substâncias classificadas pelas agências internacionais como agentes carcinogênicos em seres humanos, o que torna extremamente relevante a realização de trabalhos de avaliação de risco (LEGAY et al., 2010; FLORENTIN; HAUTEMANIÈRE; HARTEMANN, 2011; USEPA, 2012; GRELLIER et al., 2015). Outros SOH potencialmente prejudiciais à saúde humana decorrentes da cloração da água também são relatados na literatura, como os ácidos haloacéticos (AHA), os haloaldeídos (HAD), as halocetonas (HK), os halofenóis (HF), as halopicrinas (HP) e o tricloronitrometano (TCNM) (LEGAY et al., 2010; BUTIÃO, 2011; GAN et al., 2013; CARDADOR; SALGUERO; GALLEGO, 2015).

De modo geral, pode-se obter a determinação de $\mathrm{SOH}$ por métodos cromatográficos e, por estarem em baixas concentrações, estes necessitam de uma etapa de pré-concentração. Podem ser isolados da matriz aquosa pelas técnicas automatizadas de headspace (HS) ou purge and trap (P\&T) ou manualmente por extração líquido-líquido (ELL), seguido pela determinação por cromatografia a gás por captura de elétrons (CG-DCE) ou cromatografia a gás acoplada à espectrometria de massas (CG-EM). Autores mencionaram também o detector de ionização por chama (DIC) para a determinação dos compostos (NIKOLAOU et al., 2002; CULEA; COZAR; RISTOIU, 2006; PAVÓN et al., 2008; ALLARD et al., 2012). A CG-DCE é a base dos métodos USEPA, porém a CG-EM tem merecido destaque por sua alta precisão e sensibilidade (CARERI; MANGIA, 2011).

A espécie Microcystis aeruginosa ocorre naturalmente em águas superficiais, com distribuição ampla no Brasil, incidindo de norte a sul no país (SANT’ANNA et al., 2008). Matthiensen, Yunes e Codd (1999),
Gomes et al. (2009), Silva et al. (2013) e Costa (2015) mencionaram a espécie em eventos de floração em concentrações que variam de $1,0 \times 10^{4}$ a $1,0 \times 10^{7}$ células. $\mathrm{mL}^{-1} \mathrm{em}$ diversas localidades do território brasileiro, além de outros estudos (STEFFEN et al., 2014; HO; MICHALAK, 2015) reportarem sua presença em escala global.

Steffen et al. (2014) e Ho e Michalak (2015) relataram que, desde meados da década de 1990, apesar de outras espécies prejudiciais também terem sido observadas no lago Erie, situado na América do Norte (Aphanizomenon spp., Anabaena spp., Cylindrospermopsis spp., e Planktothrix spp.), a Microcystis aeruginosa tem sido a espécie tóxica dominante, o que mostra a relevância do estudo do gênero, já que é associada a inúmeros casos de floração. Essa espécie é conhecida pela liberação de microcistinas hepatotóxicas e por sua flutuabilidade, estratégia para competir com outras espécies. A microcistina é uma cianotoxina danosa ao fígado e potencialmente prejudicial ao ser humano (CHORUS; BARTRAM, 1999). Segundo Huang et al. (2009) e Zamyadi et al. (2013), a cianobactéria pode ser precursora da formação de TAM durante os processos de tratamento da água quando se utiliza o cloro.

O desenvolvimento de pesquisas que utilizem método analítico simples justifica-se pelo baixo custo para determinação de TAM (TCM, BDCM, DBCM e TBM) em amostras cloradas contendo cianobactérias. Dessa forma, o objetivo desta pesquisa foi desenvolver e validar um método com a técnica CG-EM juntamente com ELL, pela simplicidade e pelos custos relativamente acessíveis, como alternativa à extração por P\&T e HS acoplada a DCE (NIKOLAOU et al., 2002; CULEA; COZAR; RISTOIU, 2006; PAVÓN et al., 2008). A espécie Microcystis aeruginosa, em condições experimentais, foi utilizada buscando avaliar o efeito da cloração com doses distintas em densidade de células que representasse um evento de floração. Os ensaios de demanda de cloro foram executados para melhor entendimento da ação de doses nas etapas de oxidação.

\section{METODOLOGIA}

\section{Validação do método de detecção de trialometanos}

A validação do método observou os seguintes parâmetros: inexistência de interferentes (seletividade), proporcionalidade entre a resposta da área de cada composto e a concentração (curva de calibração e linearidade), determinação dos limites de detecção (LD) e quantificação (LQ), exatidão e precisão (RIBANI et al., 2004; ZANCHETTA; PENA; GONÇALVES, 2015; INMETRO, 2016; BRASIL, 2017b).

\section{Reagentes e materiais}

Reagentes de alto grau analítico foram utilizados, assim como água ultrapura obtida por meio do sistema de purificação de água da Thermo Fisher. Adquiriram-se padrões certificados de pureza cromatográfica de TAM (TCM, BDCM, DBCM e TBM) (4M8140-U, TraceCERT $\left.{ }^{\circledR}\right)$ e 
do padrão interno fluorbenzeno (CRM48943, TraceCERT ${ }^{\circledR}$ ), ambos de concentração $2.000 \mu \mathrm{g} \cdot \mathrm{mL}^{-1}$ em metanol da marca Sigma. Para a ELL, metanol (646377) e metil-terc-butílico éter (MTBE) (34875), também da Sigma, e sulfato de sódio $\left(\mathrm{Na}_{2} \mathrm{SO}_{4}\right)$ foram empregados.

\section{Otimização das condições de extração e elaboração da curva de calibração}

As condições de extração para a determinação cromatográfica basearam-se nas recomendações do método 551.1 da USEPA (1995b), com modificações (uso da EM em substituição ao DCE) como proposta de se obter um método sensível e, ao mesmo tempo, simples. Inicialmente, foram ajustadas seis rampas para a obtenção da melhor corrida cromatográfica por injeção a partir de uma extração na concentração $200 \mu \mathrm{g} . \mathrm{L}^{-1}$, tomando por referência valores monitorados por Nikolaou et al. (2002), Culea, Cozar e Ristoiu (2006) e Cardador, Salguero e Gallego (2015) para a identificação da presença dos TAM.

Após a identificação das relações massa/carga $\left(\mathrm{m} \cdot \mathrm{z}^{-1}\right)$, procedeu-se com a corrida (modo total ion current - TIC) para a separação do TCM, BDCM, DBCM e TBM (modo selected ion recording - SIR) nas rampas mencionadas, com melhor condição obtida descrita no item Instrumentação e condições cromatográficas. Para construção da curva de calibração, bem como no estudo da linearidade do método, foram preparadas diluições nas concentrações $1,2,5,10,20,40,60,80,150$ e $200 \mu \mathrm{g} . \mathrm{L}^{-1}$, além de 400, 600, 800 e $1000 \mu \mathrm{g} . \mathrm{L}^{-1}$ em $50 \mathrm{~mL}$ de água ultrapura para os elementos TCM, BDCM, DBCM e TBM.

As diluições foram rapidamente coletadas e inseridas em frascos do tipo âmbar de $100 \mathrm{~mL}$ com $50 \mu \mathrm{L}$ de padrão interno fluorbenzeno (diluído), $4 \mathrm{~mL}$ de MTBE (mantido a $4^{\circ} \mathrm{C}$ ) e aproximadamente $1 \mathrm{~g}$ de sulfato de sódio puro (agente secante da fase orgânica). $O$ frasco âmbar escuro foi manualmente agitado vigorosamente por 5 minutos e imediatamente acondicionado em freezer a $-4^{\circ} \mathrm{C}$. Duas fases, bem definidas, formaram-se dentro do frasco âmbar: a orgânica superior, chamada de extrato, e a inferior, denominada de fase aquosa. A fase orgânica foi retirada e transferida para um frasco tipo vial de $2 \mathrm{~mL}$, foi acondicionada em freezer a $-4^{\circ} \mathrm{C}$ e foi conservada para análise (validade analítica de 14 dias) em CG-EM para a verificação dos parâmetros de validação.

\section{Instrumentação e condições cromatográficas}

Utilizou-se um sistema de CG-EM, o CLARUS 680 SQ 8 PerkinElmer (EUA), para as análises, empregando-se os padrões dos TAM para a detecção.

As condições otimizadas para o CG foram $2 \mu \mathrm{L}$ de amostra injetada no modo splitless separados por coluna capilar (ZEBRON ZB-5MS; $30 \mathrm{~m}$ length $\times 0,25 \mathrm{~mm}$ diameter $\times 0,25 \mu \mathrm{m}$ film; fase G27 - 5\% Polysilarylene e $95 \%$ Polydimethylsiloxane). A temperatura do injetor foi $200^{\circ} \mathrm{C}$, o gás de arraste foi o hélio, de grau de pureza 99,9995\% e fluxo a 1,0 mL.min ${ }^{-1}$ (fluxo constante). $\mathrm{O}$ forno foi programado isotermicamente a $35^{\circ} \mathrm{C}$ por 1 minuto e, em seguida, aumentado para $40^{\circ} \mathrm{C}$ a uma velocidade de $1^{\circ} \mathrm{C} \cdot \mathrm{min}^{-1}$, permanecendo a essa temperatura por 1 minuto, seguido de aquecimento de $30^{\circ} \mathrm{C} \cdot \mathrm{min}^{-1}$ até $200^{\circ} \mathrm{C}$.

O EM foi usado no modo de impacto de elétrons (EI+). O tempo de corte do solvente foi de 3 minutos, e a velocidade de aquisição foi de 0,35 s.scan ${ }^{-1}$, intervalo de leitura m. $\mathrm{Z}^{-1}$ de 45 a 260 u.m.a. e modo de ionização de impacto eletrônico $70 \mathrm{eV}$. A temperatura da linha de transferência foi de $200^{\circ} \mathrm{C}$, e a do trap, de $180^{\circ} \mathrm{C}$.

\section{Aplicação do método de detecção de trialometanos na cloração de cianobactérias}

\section{Manutenção, cultivo e contagens de células}

O cultivo das cianobactérias, assim como as análises cromatográficas, foi realizada nos Laboratórios Multiusuário e de Contaminantes do Instituto de Ciência, Engenharia e Tecnologia (ICET), pertencente à Universidade Federal dos Vales do Jequitinhonha e Mucuri (UFVJM), campus Mucuri, em Teófilo Otoni (MG). A cepa tóxica de Microcystis aeruginosa foi obtida no Laboratório de Ficologia (ICB-UFMG) e foi cultivada conforme orientação de CETESB (2013) e Jacinavicius et al. (2013), sob as seguintes condições: temperatura $23 \pm 2^{\circ} \mathrm{C}$, irradiância 40-50 $\mu \mathrm{mol}$ fótons. $\mathrm{m}^{-2} \cdot \mathrm{s}^{-1} \mathrm{e}$ fotoperíodo de 14-10 horas claro-escuro, conduzidas em erlenmeyers replicados de $500 \mathrm{~mL}$ e $1 \mathrm{~L}$.

Foi utilizado meio de cultivo concentrado ASM-1, preparado com água destilada e esterilizado para evitar quaisquer contaminações e interferências nos resultados. Após o preparo, este foi armazenado a $-4^{\circ} \mathrm{C}$. Foi ajustado o $\mathrm{pH}$ do meio para 7,4 , com soluções de $\mathrm{HCl}$ (ácido clorídrico) $1 \mathrm{M}$ para acidificar o meio e de $\mathrm{NaOH}$ (hidróxido de sódio) $1 \mathrm{M}$ para elevar o $\mathrm{pH}$, quando necessário.

\section{Amostragem e extração nas amostras}

A densidade de $1,0 \times 10^{6}$ células. $\mathrm{mL}^{-1}$, obtida durante a fase exponencial de crescimento (período de 20-25 dias), foi utilizada para os ensaios por estar associada a eventos de floração conforme descrito anteriormente. A contagem das células foi feita utilizando microscópio óptico, com auxílio da câmera de Neubauer, recomendada para quantificação de células fitoplanctônicas e de cianobactérias (JACINAVICIUS et al., 2013; BRASIL, 2015).

O cloro gasoso foi escolhido como oxidante por ser usualmente utilizado nas estações de grande porte, uma vez que reduz a utilização de espaços para armazenamento dentro da estação e minimiza problemas logísticos no abastecimento, comparando-se aos hipocloritos de cálcio ou sódio (DI BERNARDO; PAZ, 2008). Ademais, ele apresenta alta eficiência na redução de gosto e odor (PASCHOALATO, 2005) e foi fornecido pela Companhia de Saneamento de Minas Gerais (COPASA), situada na região do Alto Mucuri, em Teófilo Otoni (MG). A real concentração de cloro da solução fornecida foi determinada por titulação, sendo o tiossulfato de sódio o titulante e o amido e o iodeto de potássio 
os indicadores. Ele foi utilizado imediatamente após o preparo para não haver perdas no processo por volatilização.

Os ensaios de demanda de cloro foram realizados em escala de bancada, na qual diferentes concentrações de cloro gasoso foram aplicadas em amostras do cultivo (com densidade de $1,0 \times 10^{6}$ células. $\mathrm{mL}^{-1}$ ). Para a etapa, erlenmeyers de $50 \mathrm{~mL}(\mathrm{n}=3)$ foram preenchidos com $50 \mathrm{~mL}$ de cultura e dosados com o oxidante nas concentrações $1 ; 1,5 ; 2,5 ; 3,75 ; 5$; 7,5; e $10 \mathrm{mg} . \mathrm{L}^{-1}$, observando-se o teor de cloro residual livre (CRL) após o término do tempo de contato de 30 minutos. As leituras de concentração e cloro residual livre foram efetuadas por método colorimétrico com adição de N-dietil-para-fenilendiamina (DPD), por meio de um comparador colorimétrico HACH Test Kit $\left(\mathrm{Cl}_{2}, 0-3,4 \mathrm{mg} \cdot \mathrm{L}^{-1} \mathrm{Cl}_{2}\right.$, modelo CN-66).

Os valores 2,5 e 5 mg. $\mathrm{L}^{-1}$ foram escolhidos para avaliar os efeitos da dose de cloro na formação de trialometanos. As condições de formação foram pH 6,5 (dentro do intervalo de 5,5 a 9,0, em que predomina a coagulação pelo mecanismo de varredura, frequentemente utilizado em ETA), temperatura $30^{\circ} \mathrm{C}$ (recorrente no Brasil) e tempo de contato de 30 minutos (formação nos primeiros minutos da cloração). As alíquotas em triplicata foram submetidas ao mesmo procedimento de extração descrito anteriormente para detecção dos trialometanos, após validação e preparação da curva de calibração. Na Figura 1, está representado o fluxograma descritivo do ensaio de oxidação com cloro gasoso da espécie Microcystis aeruginosa.

\section{RESULTADOS E DISCUSSÃO}

\section{Parâmetros de validação analítica}

A sequência TCM, BDCM, DBCM e TBM, obtida por meio do cromatograma da condição descrita na metodologia (Figura 2), é explicada pelo fato de os compostos apresentarem características químicas distintas. O TCM, por apresentar menor massa molar, é detectado na coluna em um menor intervalo de tempo, enquanto o TBM é o último dos compostos por apresentar massa molar maior que os outros TAM. Outras características, como ponto de ebulição e fusão, validam o resultado, como descrito na Tabela 1. Após a otimização da rampa mencionada, obtiveram-se os valores dos parâmetros da validação, postados nas Tabelas 2 e 3.

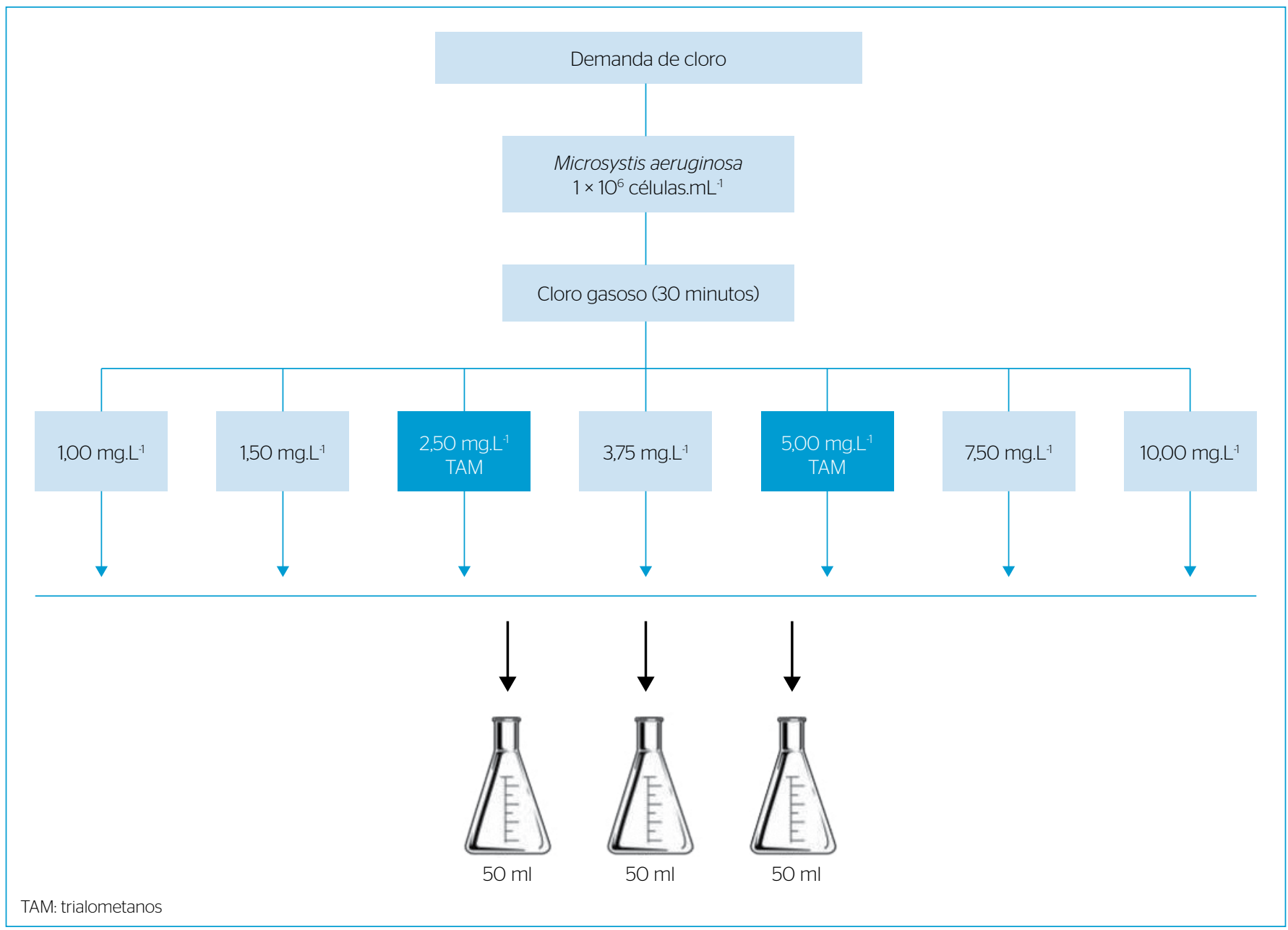

Figura 1 - Demanda de cloro utilizado nos ensaios de determinação de trialometanos na cloração da Microcystis aeruginosa. 


\section{Curva analítica e linearidade}

Pelos resultados da regressão linear $(\mathrm{Y}=\mathrm{aX}+\mathrm{b})$ obtidos pelas curvas de calibração ( $\mathrm{Y}$ como a sensibilidade ou intensidade $\mathrm{X}$ como a concentração de cada TAM no CG-EM), observaram-se (Tabela 2) respostas lineares e correlações acima do que preconiza os órgãos certificadores para as faixas de concentrações estudadas $(p=10$ en $=3)$. Brasil (2017b) recomenda coeficiente de correlação igual a 0,99, e o INMETRO (2016), um valor acima de 0,90. Foi verificado que houve boa linearidade quando foram utilizadas concentrações de 1 até $1000 \mu \mathrm{g} . \mathrm{L}^{-1}$, também com correlações superiores sugeridas por INMETRO (2016) e Brasil (2017b). O método forneceu resultados diretamente proporcionais à concentração dos TAM dentro da faixa de aplicação.
Tabela 1 - m. $z^{-1}$, tempo de retenção, massa molar, ponto de ebulição e fusão dos trialometanos.

\begin{tabular}{l|c|c|c|c|c} 
Trialometano & $\mathrm{m} \cdot \mathrm{z}^{1}$ & $\begin{array}{c}\text { Tempo de } \\
\text { retenção } \\
(\mathrm{min.})\end{array}$ & $\begin{array}{c}\text { Massa } \\
\text { molar } \\
\left(\mathrm{g} \cdot \mathrm{mol}^{-1}\right)\end{array}$ & $\begin{array}{c}\text { Ponto de } \\
\text { ebulição } \\
\left({ }^{\circ} \mathrm{C}\right)\end{array}$ & $\begin{array}{c}\text { Ponto } \\
\text { de fusão } \\
\left({ }^{\circ} \mathrm{C}\right)\end{array}$ \\
\hline TCM & $83 ; 85$ & 3,15 & 119,38 & 61,20 & $-63,50$ \\
\hline BDCM & $83 ; 85 ; 127$ & 4,37 & 163,80 & 90 & -57 \\
\hline DBCM & $127 ; 129$ & 7,17 & 242,72 & 130 & 38 \\
\hline TBM & $175 ; 252$ & 9,02 & 252,73 & 149,10 & 8 \\
\hline
\end{tabular}

TCM: triclorometano; BDCM: bromodiclorometano; DBCM: dibromoclorometano; TBM: tribromometano.

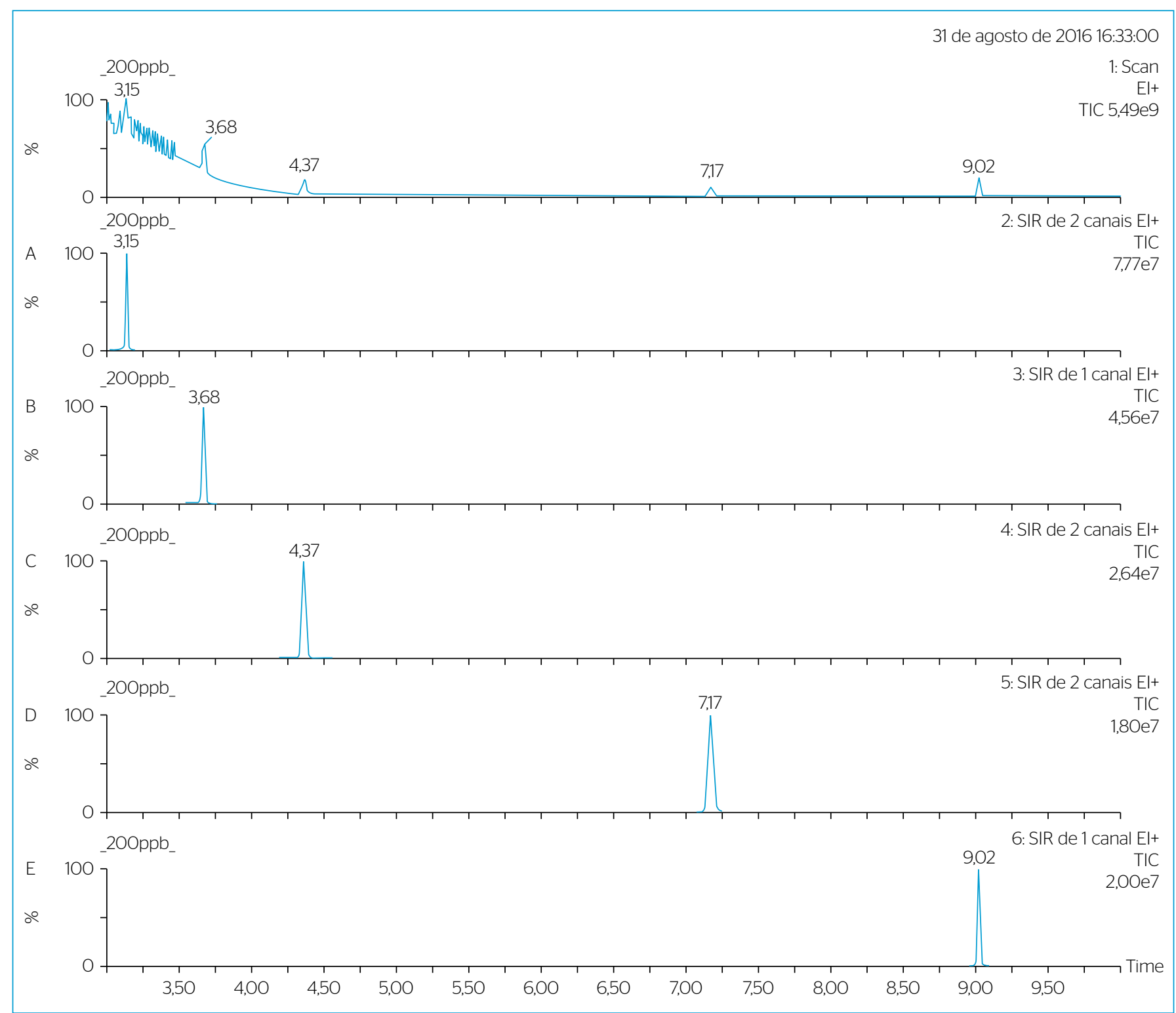

Figura 2 - Cromatograma modo total ion current e selected ion recording da otimização da rampa dos trialometanos, inclusive o padrão interno fluorbenzeno, obtido por injeção a partir de uma extração na concentração $200 \mu \mathrm{g} \cdot \mathrm{L}^{-1}$. (A) selected ion recording 2, triclorometano; (B) selected ion recording 3, fluorbenzeno; (C) selected ion recording 4, bromodiclorometano; (D) selected ion recording 5 , dibromoclorometano; (E) selected ion recording 6 , tribromometano. 


\section{Precisão}

A precisão foi obtida por meio da estimativa do desvio padrão relativo (RSD, na sigla em inglês) em função de repetitividade e precisão intermediária. Em métodos de análise de traços ou impurezas, são aceitos RSD de até $20 \%$, dependendo da complexidade da amostra (RIBANI et al., 2004; INMETRO, 2016; BRASIL, 2017b). A repetitividade foi avaliada por injeções em um mesmo dia, em amostra de $200 \mu \mathrm{g} \cdot \mathrm{L}^{-1}(\mathrm{n}=6)$. Conforme constatado na Tabela 2 , foi possível observar a similaridade dos resultados e concluir que os RSD foram inferiores a $2 \%$. Para a precisão intermediária, avaliada por injeções em dias distintos $(n=5)$ em concentração idêntica à repetitividade, os RSD foram inferiores a 7\%, também dentro do limite aceitável (20\%) (Tabela 2).

\section{Limite de detecção e limite de quantificação}

Os limites de detecção (LD) e quantificação (LQ) foram obtidos pelo método baseado em parâmetros da curva analítica (RIBANI et al., 2004; INMETRO, 2016; BRASIL, 2017b). O LD ( $\mathrm{n}=10$ ) foi calculado com base na relação de 3,3 vezes do desvio padrão do branco dividido pelo coeficiente angular da curva analítica, e o LQ $(n=10)$ foi a relação de 10 vezes também do desvio padrão do branco dividido pelo coeficiente angular da curva analítica (1-200 $\left.\mu \mathrm{g} . \mathrm{L}^{-1}\right)$. Conforme observado na Tabela 2, o método é capaz de detectar e quantificar baixas concentrações, permitindo maior confiabilidade nos resultados. Ribani et al. (2004) o recomendam por ser estatisticamente mais confiável.

\section{Exatidão}

Os resultados obtidos através de valores de recuperação $(n=3)$ em quatro concentrações diferentes $\left(20,80,150\right.$ e $\left.200 \mu \mathrm{g} . \mathrm{L}^{-1}\right)$ variaram de 93,01 a 110,08\% (Tabela 3). Os intervalos aceitáveis de recuperação para análise de resíduos geralmente estão entre 70 e $120 \%$, com precisão de até $20 \%$. Com a curva de 1-200 $\mu \mathrm{g} . \mathrm{L}^{-1}$, foi possível estimar os valores esperados em função da resposta das injeções nas concentrações sugeridas, sendo próximos aos de interesse. Os RSD calculados também foram satisfatórios (menores que 5\%). Ressalta-se que foram utilizados materiais de referência certificados $\left(\right.$ TraceCERT $\left.^{\circledR}\right)$ para garantia da qualidade no processo de validação analítica (RIBANI et al., 2004; INMETRO, 2016; BRASIL, 2017b).

\section{Seletividade}

A seletividade foi definida comparando a matriz da substância (padrão) com a adição do composto 2,2-dicloropropiônico (47656-U, TraceCERT ${ }^{\circledR}$ ). Como não houve interferência no tempo de retenção dos TAM,

Tabela 3 - Parâmetros de validação dos trialometanos: exatidão e ensaios de recuperação.

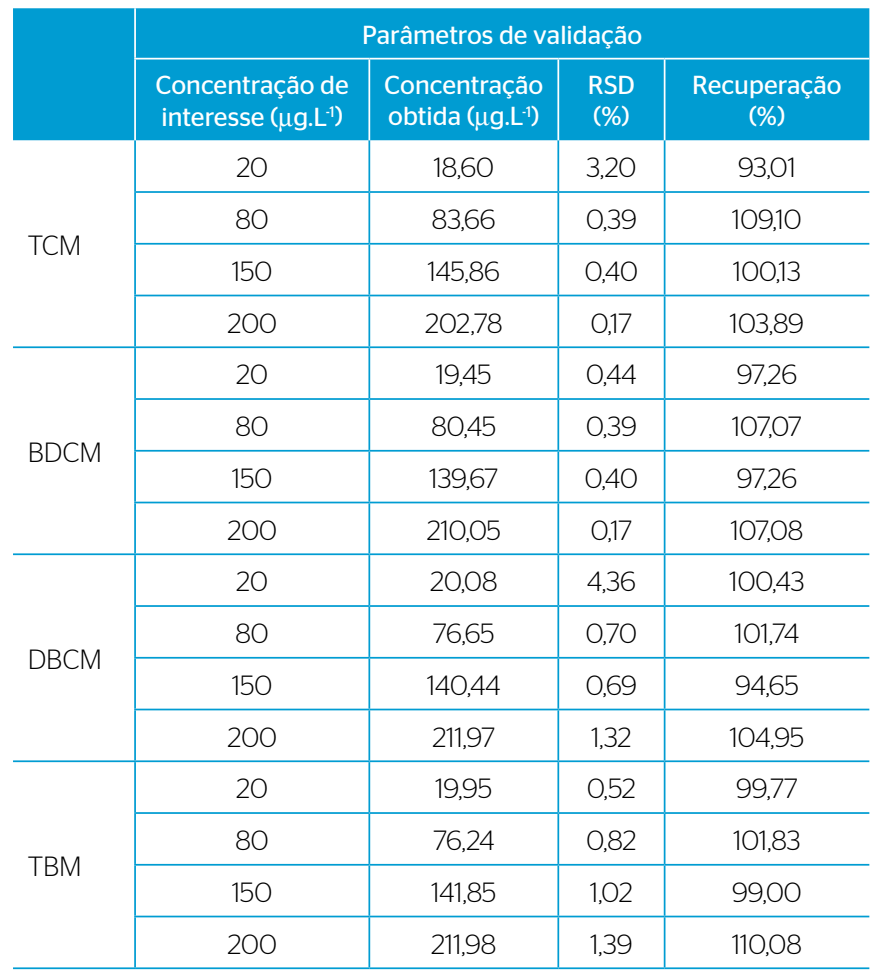

RSD: relative standard deviation; TCM: triclorometano; BDCM: bromodiclorometano; DBCM: dibromoclorometano; TBM: tribromometano.

Tabela 2 - Parâmetros de validação dos trialometanos: coeficiente de correlação e determinação, precisão, limite de detecção e quantificação.

\begin{tabular}{|c|c|c|c|c|}
\hline Parâmetros de validação & TCM & $\mathrm{BDCM}$ & DBCM & TBM \\
\hline Curva de calibração $\left(1-200 \mu \mathrm{g} . \mathrm{L}^{-1}\right)$ & $Y=1,114+0,607 * X$ & $Y=-1,989+0,702 * X$ & $Y=-3,417+0,716 * X$ & $Y=-1,997+0,374 * X$ \\
\hline Curva de calibração (1-1000 $\left.\mu \mathrm{g} \cdot \mathrm{L}^{-1}\right)$ & $Y=-0,439+0,600 * X$ & $Y=-6,161+0,707 * X$ & $Y=-9,91+0,755 * X$ & $Y=-3,278+0,366 * X$ \\
\hline $\begin{array}{l}\text { Coeficiente de correlação (r) /determinação }\left(r^{2}\right) \\
\left(1-200 \mu \mathrm{g} \cdot \mathrm{L}^{-1}\right)\end{array}$ & 0,9992 / 0,9985 & 0,9967 / 0,9934 & 0,9950 / 0,9900 & 0,9950 / 0,9901 \\
\hline $\begin{array}{l}\text { Coeficiente de correlação }(r) \text { /determinação }\left(r^{2}\right) \\
\left(1-1000 \mu g \cdot L^{-1}\right)\end{array}$ & 0,9979 / 0,9959 & 0,9954 / 0,9909 & 0,9945 / 0,9900 & 0,9957 / 0,9914 \\
\hline Precisão intermediária RSD (\%) (200 $\left.\mu \mathrm{g} . \mathrm{L}^{-1}\right)$ & 6,19 & 3,70 & 2,23 & 1,98 \\
\hline Precisão de repetitividade RSD (\%) (200 $\left.\mu \mathrm{g} . \mathrm{L}^{-1}\right)$ & 1,87 & 0,17 & 1,32 & 1,39 \\
\hline Limite de detecção (LD - $\left.\mu \mathrm{g} \mathrm{L}^{-1}\right)\left(1-200 \mu \mathrm{g} . \mathrm{L}^{-1}\right)$ & 0,13 & 0,02 & 0,02 & 0,01 \\
\hline Limite de quantificação (LQ $\left.\mu \mathrm{g} \cdot \mathrm{L}^{-1}\right)\left(1-200 \mu \mathrm{g} . \mathrm{L}^{-1}\right)$ & 0,40 & 0,07 & 0,07 & 0,05 \\
\hline
\end{tabular}

TCM: triclorometano; BDCM: bromodiclorometano; DBCM: dibromoclorometano; TBM: tribromometano; RSD: relative standard deviation. 
manteve-se a característica de estar bem separada durante os cromatogramas, o que indica a seletividade da metodologia proposta.

\section{Comparação dos resultados analíticos da validação com trabalhos da literatura}

Na literatura (USEPA, 1995a; 1995b; APHA; AWWA; WEF, 2012), encontra-se descrito um número significativo de metodologias analíticas para a quantificação de TAM, conforme Tabela 4 .

Diferentes tempos de retenção podem ser explicados pelo uso de diferentes colunas. Nikolaou et al. (2002) usaram a coluna tipo HP-VOC $(60,00 \mathrm{~m} \times 0,32 \mathrm{~mm} \times 1,80 \mu \mathrm{m})$ e Culea, Cozar e Ristoiu (2006) utilizaram a mesma deste trabalho, porém de outra fabricante (RTX-5MS; 30,00 m $\times 0,25 \mathrm{~mm} \times 0,25 \mu \mathrm{m})$. Outra justificativa da variação de eficiência da separação e do desempenho obtidos neste trabalho pode estar relacionada com o gás utilizado como fase móvel. Segundo Grob (1995), o hélio possui ótima flexibilidade na escolha da vazão (velocidade linear) sem que haja perda apreciável de eficiência e é inerte se comparado ao hidrogênio.

Outro detalhe importante é o uso do padrão interno, que apresentou diferentes comportamentos quando comparado com resultados de outros trabalhos pesquisados (CULEA; COZAR; RISTOIU, 2006). O padrão fluorbenzeno apresenta custo menor que o halotano, utilizado por Culea, Cozar e Ristoiu (2006) em ELL-CG-EM, e para o diclorometano, usado por Paschoalato (2005) e Butião (2011) em ELL-CG-DCE. Assim sendo, estudos com padrões internos com comprovada eficiência, aliados a valores mais acessíveis, proporcionam vantagens em países que têm obrigatoriedade de monitoramento.

Culea, Cozar e Ristoiu (2006) determinaram as concentrações $20,40,60,80,100,200,300$ e $400 \mu g . L^{-1}$ dos 4 TAM por ELL-CG-EM usando o padrão interno halotano. Os autores compararam os resultados com as técnicas P\&T-CG-EM e HS-CG-EM e, apesar de indicarem a última como a mais sensível, a ELL-CG-EM se mostrou eficiente. Em comparação com este estudo, obtiveram-se melhores coeficientes de regressão e LD (abaixo de $1 \mu \mathrm{g} . \mathrm{L}^{-1}$ ), sendo que alguns estudos por ELL (BRAGA et al., 2004; CULEA; COZAR; RISTOIU, 2006) começam a detectar, após $20 \mu \mathrm{g} . \mathrm{L}^{-1}$, a recomendação de uso de outras técnicas de extração (P\&T e HS) para maior confiabilidade. Apesar de o HS e P\&T constarem entre as escolhas

Tabela 4 - Métodos cromatográficos para detecção de trialometanos.

\begin{tabular}{l|c|c}
\hline Método & Concentração & Separação e detecção \\
\hline USEPA 524.2 & P\&T & CG-EM \\
\hline USEPA 551.1 & ELL & CG-DCE \\
\hline APHA 6200 & P\&T & CG-EM \\
\hline APHA 6210 & P\&T & CG-EM \\
\hline APHA 6232 & ELL & CG-DCE \\
\hline
\end{tabular}

P\&T: purge and trap; ELL: extração líquido-líquido; CG-EM: cromatografia a gás acoplada à espectrometria de massas; CG-DCE: cromatografia a gás por captura de elétrons. preferenciais devido à sua automação, outras técnicas, tais como ELL associada ao CG-EM, podem oferecer excelente desempenho com um investimento menor.

\section{Ensaios de demanda de cloro}

A Tabela 5 apresenta os resultados de CRL obtidos dos três ensaios nas doses variantes entre 1 e $10 \mathrm{mg} . \mathrm{L}^{-1}$.

Esses resultados demonstraram alto consumo de cloro e baixos valores de CRL, e estudos como o de Plummer e Edzwald (2001) afirmaram que o grau de ruptura da célula está diretamente relacionado à morfologia celular e à taxa de célula/cloro. Culturas de cianobactérias, segundo Ma et al. (2012), contêm uma ampla gama de compostos, como a matéria orgânica extracelular (MOE), liberada devido à autólise das células, e a matéria orgânica intracelular (MOI), geralmente liberada por lise comprometida ou morte celular.

Segundo Fan (2012), a lise celular pode levar a uma liberação de toxinas ligada à célula e ao carbono orgânico dissolvido (COD), o que explica uma demanda significativa de cloro. De acordo com Ma et al. (2012), após o cloro penetrar nas células, reage com diferentes materiais, tais como membranas celulares e substâncias intracelulares, aumentando o consumo e, consequentemente, ampliando as chances de formação de $\mathrm{SOH}$.

\section{Análise de formação de trialometanos após cloração com Microcystis aeruginosa}

A Tabela 6 apresenta os resultados das análises de TAM $(n=3)$ em amostras com a espécie de cianobactéria Microcystis aeruginosa.

O TCM foi detectado em todas as amostras cloradas, associado única e exclusivamente à cianobactéria clorada e possivelmente aos metabólitos produzidos pela espécie, já que foram cultivadas em água ultrapura, apenas com o meio ASM-1 e sem contaminantes externos. A não ocorrência de espécies bromadas pode ser justificada pela provável ausência de íons brometo, que podem participar da etapa de halogenação da sequência de reação dos compostos TAM de modo mais eficaz do que o cloro. Yang, Guo e Shen (2011) observaram que

Tabela 5 - Resultados de cloro residual livre para as doses aplicadas de cloro gasoso.

\begin{tabular}{l|c|c|c|c|c|c}
$\begin{array}{l}\text { Oxidante } \\
\text { químico }\end{array}$ & $\begin{array}{c}\text { Dose } \\
\left(\mathrm{mg} \cdot \mathrm{L}^{-1}\right)\end{array}$ & $\begin{array}{c}\text { Ensaio 1 } \\
1,0 \times 10^{6} \\
30 \mathrm{~min} .\end{array}$ & $\begin{array}{c}\text { Ensaio 2 } \\
1,0 \times 10^{6} \\
30 \mathrm{~min} .\end{array}$ & $\begin{array}{c}\text { Ensaio 3 } \\
1,0 \times 10^{6} \\
30 \mathrm{~min} .\end{array}$ & $\begin{array}{c}\text { Média } \\
1,0 \times 10^{6} \\
30 \mathrm{~min} .\end{array}$ & $\begin{array}{c}\text { Desvio } \\
\text { padrão } \\
30 \mathrm{~min} .\end{array}$ \\
\cline { 2 - 7 } & 1,00 & 0,10 & 0,10 & 0,10 & 0,10 & 0,00 \\
\cline { 2 - 7 } & 1,50 & 0,10 & 0,10 & 0,10 & 0,10 & 0,00 \\
\cline { 2 - 7 } $\begin{array}{l}\text { Cloro } \\
\text { gasoso }\end{array}$ & 2,50 & 0,10 & 0,10 & 0,10 & 0,10 & 0,00 \\
\cline { 2 - 7 } & 3,75 & 0,10 & 0,20 & 0,10 & 0,10 & 0,06 \\
\cline { 2 - 7 } & 5,0 & 0,10 & 0,20 & 0,10 & 0,10 & 0,06 \\
\cline { 2 - 7 } & 7,50 & 0,10 & 0,50 & 0,20 & 0,30 & 0,21 \\
\cline { 2 - 7 } & 10,00 & 0,30 & 0,50 & 0,30 & 0,40 & 0,12 \\
\hline
\end{tabular}


Tabela 6 - Resultados de trialometanos obtidos a partir da cloração com cloro gasoso $\left(\mathrm{Cl}_{2}\right)$.

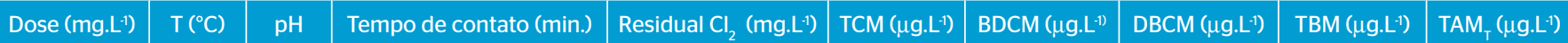

\begin{tabular}{l|l|l|l|l|l|l|l|l|l}
2,5 & 30 & 6,5 & 30 & 0,1 & 32,93 & $<$ LD & ND & ND & 32,93 \\
\hline 5,0 & 30 & 6,5 & 30 & 0,2 & 63,12 & $<$ LD & ND & ND & 63,12 \\
\hline
\end{tabular}

T: temperatura; TCM: triclorometano; BDCM: bromodiclorometano; DBCM: dibromoclorometano; TBM: tribromometano; TAM: trialometanos; LD: abaixo do limite de detecção; ND: não detectado.

a presença de bromo propiciou a formação de espécies bromadas na cloração de amostras contendo o gênero Microcystis.

$\mathrm{O} C O D$ ligado à célula de cianobactérias pode ser fonte significativa de precursores de SOH (PLUMMER; EDZWALD, 2001; HONG et al., 2008; HUANG et al., 2009). A MOE e a MOI, segundo Bond et al. (2009), Huang et al. (2009) e Zamyadi et al. (2013), podem contribuir para a formação dos TAM devido à reação do material lançado com cloro. Visto que não foi feita a separação entre células com a MOI e a MOE, as concentrações de TAM formados nos estudos podem também estar ligadas a esses compostos.

Os resultados de Zamyadi et al. (2013) em amostras contendo a espécie Microcystis aeruginosa com valores próximos ao que foi utilizado no experimento apresentaram modestas concentrações de TAM em relação a este estudo. A cloração de densas suspensões $\left(5,0 \times 10^{5}\right.$ células. $\mathrm{mL}^{-1}$; 5,5 mg.L.-1 de cloro gasoso; $\mathrm{pH} 8$ ) em água pura com o tempo de contato de 24 horas ocasionou concentrações modestas de TAM (13 $\left.\mu \mathrm{g} \cdot \mathrm{L}^{-1}\right)$. A contradição pode ser explicada pela provável diferença entre a composição da matéria orgânica dissolvida e a idade do cultivo. Como esperado, em amostras sob as mesmas condições, quanto maior a dose aplicada, maior será o consumo de oxidante e, consequentemente, maiores serão as concentrações de subprodutos formados (RODRIGUES, 2014), o que justifica os valores encontrados com a aplicação das doses neste estudo e em outros trabalhos (RODRÍGUEZ et al., 2007).

\section{CONCLUSÃO}

A validação por ELL-CG-EM mostrou-se satisfatória ao utilizar os parâmetros discorridos neste artigo, sendo, portanto, viável a aplicação em ETA com menos recursos financeiros do que o exigido pelas técnicas P\&T-CG-EM e HS-CG-EM. O detector EM mostrou-se sensível, com baixas interferências (ruídos) ao redor da posição de detecção dos TAM, tornando-se alternativa ao DCE. Pode-se concluir que cada fabricante poderá fornecer, em uma mesma condição, cromatogramas com tempos de retenção diferentes com variações nas fases em que serão construídas. Uma coluna do tipo DB-5MS é semelhante à de outros fabricantes, como RTX-5MS ou HP-5MS, as fases são semelhantes e todas são compatíveis com cromatógrafos em fase gasosa.

Procedeu-se a aplicação do método em amostras reais cloradas contendo Microcystis aeruginosa, sendo detectada a presença significativa do TCM, mostrando que a técnica ELL-CG-EM pode ser replicada para a extração e detecção de TAM após a cloração de outras espécies tóxicas. A contradição de valores na literatura de formação de TAM e a alta demanda de cloro nos ensaios de doses associados à baixa remoção de células podem estar relacionadas à composição heterogênea da fração dissolvida das amostras cloradas. O método desenvolvido e validado apresentou-se eficiente na aplicação em amostras reais, com boas sensibilidade e precisão e ótima resposta analítica, podendo ser implantado em processos de monitoramento de TAM em ETA.

\section{AGRADECIMENTOS}

Os autores agradecem à Fundação de Amparo à Pesquisa do Estado de Minas Gerais (FAPEMIG), as bolsas de iniciação científica vinculadas à pesquisa; e à Fundação Nacional de Saúde (FUNASA), o apoio financeiro.

\section{REFERÊNCIAS}

ALLARD, S.; CHARROIS, J.W.A.; JOLL, C.A.; HEITZ, A. (2O12) Simultaneous analysis of 10 trihalomethanes at nanogram per liter levels in water using solid-phase microextraction and gas chromatography mass-spectrometry. Journal of Chromatography A, v. 1238, p. 15-21. https://doi.org/10.1016/j. chroma.2012.03.020

AMERICAN PUBLIC HEALTH ASSOCIATION (APHA); AMERICAN WATER WORKS ASSOCIATION (AWWA); WATER ENVIRONMENTAL
FEDERATION (WEF). (2012) Standard methods for the examination of water and wastewater. 22. ed. Washington, D.C.: APHA; AWWA; WEF. $1496 \mathrm{p}$.

BOND, T.; HENRIET, O.; GOSLAN, E.H.; PARSONS, S.A.; JEFFERSON, B. (2009) Disinfection byproduct formation and fractionation behavior of natural organic matter surrogates. Environmental Science and Technology, v. 43, n. 15, p. 5982-5989. https://doi. org/10.1021/es900686p 
BRAGA, F.M.G; ARAÚJO, J.C.; SALES, M.V.; NASCIMENTO, R.F.; PÁDUA, V.L. (2004) Diagnóstico de ocorrência de trialometanos na rede de abastecimento de água de Fortaleza- CE, Brasil. In: CONGRESO INTERAMERICANO DE INGENIERIA SANITARIA Y AMBIENTAL, 29., San Juan, Porto Rico. Anais... p. 1-14.

BRASIL. (2015) Cianobactérias/cianotoxinas: procedimentos de coleta, preservação e análise. Brasília: Secretaria de Vigilância em Saúde, Departamento de Vigilância em Saúde Ambiental e Saúde do Trabalhador, Ministério da Saúde.

BRASIL. (2017a) Portaria de consolidação no 5, de 28 de setembro de 2017. Consolidação das normas sobre as ações e os serviços de saúde do Sistema Único de Saúde. Brasília: Ministério da Saúde.

BRASIL. (2017b) Resolução da Diretoria Colegiada RDC no 166, de 24 de julho de 2017. Dispõe sobre a validação de métodos analíticos e dá outras providências. Brasília: Agência Nacional de Vigilância Sanitária (ANVISA), Ministério da Saúde.

BUTIÃO, D.F.(2011) Formação de subprodutos orgânicos halogenados da desinfecção em águas de piscinas. 89f. Dissertação (Mestrado em Tecnologia Ambiental) - Centro de Ciências Exatas, Naturais e Tecnológicas. Universidade de Ribeirão Preto, Ribeirão Preto.

CARDADOR, M.J.; SALGUERO, J.F.; GALLEGO, M. (2O15) Simultaneous quantification of trihalomethanes and haloacetic acids in cheese by on-line static headspace gas chromatographymass spectrometry. Journal of Chromatography A, v. 1408, p. 22-29. https://doi.org/10.1016/j.chroma.2015.07.007

CARERI, M.; MANGIA, A. (2011) Trends in analytical atomic and molecular mass spectrometry in biology and the life sciences. Analytical and Bioanalytical Chemistry, v. 399, n. 8, p. 2585-2595. https://doi.org/10.1007/s00216-010-4585-8

CHORUS, I.; BARTRAM, J. (1999) Toxic cyanobacteria in water: a guide to their public health consequences, monitoring and management. Londres: World Health Organization.

COMPANHIA AMBIENTAL DO ESTADO DE SÃO PAULO (CETESB). (2013) Manual de Cianobactérias Platônicas: Legislação, Orientações para o Monitoramento e Aspectos Ambientais. São Paulo: Cetesb.

COMUNIDAD EUROPEA. (1998) DIRECTIVA 98/83/CE del consejo de 3 de noviembre de 1998 relativa a la calidad de las aguas destinadas al consumo humano. Diario Oficial de las Comunidades Europeas, n. L 330 .

CORAL, L.A.; ZAMYADI, A.; BARBEAU, B.; BASSETI, F.J.; LAPOLLI, F.R.; PRÉVOST, M. (2O13) Oxidation of Microcystis aeruginosa and Anabaena flos-aquae by ozone: Impacts on cell integrity and chlorination by-product formation. Water Research, v. 47, n. 9, p. 2983-2994. https://doi.org/10.1016/j.watres.2013.03.012

COSTA, N.B. da. (2015) Dinâmica temporal das cianobactérias em um reservatório urbano hipereutrófico: uma abordagem morfológica e molecular. 74f. Dissertação (Mestrado em Ecologia, Conservação e Manejo da Vida Silvestre) - Instituto de Ciências Biológicas, Universidade Federal de Minas Gerais, Belo Horizonte.
CULEA, M: COZAR, O: RISTOIU, D. (2006) Methods validation for the determination of trihalomethanes in drinking water. Journal of Mass Spectrometry, v. 41, n. 12, p. 1594-1597. http://dx.doi.org/10.1002/jms.1149

DI BERNARDO, L.; PAZ, L.P.S. (2008) Seleção de tecnologias de tratamento de água. São Carlos: Ed. LDIBE. v. 2. 682 p.

FAN, Y. (2012) Chlorination of toxic cyanobacterial cells and their associated toxins. 123f. Mémoire de maîtrise (Maîtrise ès Sciences Appliquées) - Génie Civil, Université de Montréal École Polytechnique de Montréal, Montréal, Canadá.

FLORENTIN, A.; HAUTEMANIËRE, A.; HARTEMANN, P. (2O11) Health effects of disinfection by-products in chlorinated swimming pools. International Journal of Hygiene and Environmental Health, v. 214, n. 6, p. 461-469. https://doi.org/10.1016/j.jijheh.2011.07.012

GAN, W.; GUO, W.; MO, J.; HE, Y.; LIU, Y; LIU, W.; LIANG, Y.; XIN, Y. (2013) The occurrence of disinfection by-products in municipal drinking water in China's Pearl River Delta and a multipathway cancer risk assessment. Science of the Total Environment, v. 447, p. 108-115. https://doi.org/10.1016/j.scitotenv.2012.12.091

GOMES, A.M.A.; SAMPAIO, P.L.; FERRÃO-FILHO, A. da S.; MAGALHÃES, V. de F; MARINHO, M.M.; OLIVEIRA, A.C.P. de; SANTOS, V.B. dos; DOMINGOS, P.; AZEVEDO, S.M.F. de. (2009) Florações de cianobactérias tóxicas em uma Lagoa Costeira hipereutrófica do Rio de Janeiro/ RJ (Brasil) e suas consequências para a saúde humana. Oecologia Australis, v. 13, n. 2, p. 329-345. http://doi.org/10.4257/oeco.2009.1302.08

GRELLIER, J.; RUSHTON, L.; BRIGGS, D.J.; NIEUWENHUIJSEN, M.J. (2015) Assessing the human health impacts of exposure to disinfection by-products - a critical review of concepts and methods. Environment International, v. 78, p. 61-81. https://doi. org/10.1016/j.envint.2015.02.003

GROB, R.L. (1995) Modern Practice of Gas Chromatography. 3. ed. Nova York: Wiley Interscience. 888 p.

HEALTH CANADA. (2017) Guidelines for Canadian drinking water quality: Summary table. Water and Air Quality Bureau, Healthy Environments and Consumer Safety Branch. Ottawa: Health Canada.

HO, J.C.; MICHALAK, A.M. (2O15) Challenges in tracking harmful algal blooms: A synthesis of evidence from Lake Erie. Journal of Great Lakes Research, v. 41, n. 2, p. 317-325. https://doi.org/10.1016/j. jglr.2015.01.001

HONG, H.C.; MAZUMDER, A.; WONG, M.H.; LIANG, Y. (2008) Yield of trihalomethanes and haloacetic acids upon chlorinating algal cells, and its prediction via algal cellular biochemical composition. Water Research, v. 42, n. 20, p. 4941-4948. https://doi.org/10.1016/j. watres.2008.09.019

HUANG, G.; GRAHAM, N.; TEMPLETON, M.R.; ZHANG, Y:; COLLINS, C:; NIEUWENHUIJSEN, M. (2009) A comparison of the role of two blue-green algae in THM and HAA formation. Water Research, v. 43, n. 12, p. 3009-3018. https://doi.org/10.1016/j.watres.2009.04.029 
INSTITUTO NACIONAL DE METROLOGIA, NORMALIZAÇÃO E QUALIDADE INDUSTRIAL (INMETRO). (2016) Revisão no 5, de agosto de 2016. Orientação sobre Validação de Métodos Analíticos: Documento de caráter orientativo. (DOQ-CGCRE-OO8). Coordenação Geral de Acreditação. Distrito Federal: Sistema Nacional de Metrologia, Normalização e Qualidade Industrial (Sismetro).

JACINAVICIUS, F.R.; G. JÚNIOR, W.A.; AZEVEDO, M.T P.; SANT'ANNA, C.L. (2013) Manual para Cultivo de Cianobactérias. São Paulo: Secretaria do Meio Ambiente do Estado de São Paulo, Instituto de Botânica, Núcleo de pesquisa em Ficologia.

LEGAY, C.; RODRIGUEZ, M.J.; SÉRODES, J.B.; LEVALLOIS, P. (2O10) Estimation of chlorination by-products presence in drinking water in epidemiological studies on adverse reproductive outcomes: A review. Science of the Total Environment, v. 408, n. 3, p. 456-472. https://doi.org/10.1016/.scitotenv.2009.10.047

LIBÂNIO, M. (2010) Fundamentos de Qualidade e Tratamento de Água. 3. ed. Campinas: Átomo. 444 p.

MA, M.; LIU, R.; LIU, H.; QU, J.; JEFFERSON, W. (2012) Effects and mechanisms of prechlorination on Microcystis aeruginosa removal by alum coagulation: Significance of the released intracellular organic matter. Separation and Purification Technology, v. 86, p. 19-25. http://dx.doi.org/10.1016/j.seppur.2011.10.015

MATTHIENSEN, A.; YUNES, J.S.; CODD, G.A. (1999) Ocorrência, Distribuição e Toxicidade de cianobactérias no estuário da Lagoa dos Patos, RS. Revista Brasileira de Biologia, v. 59, n. 3, p. 361-376. http://dx.doi.org/10.1590/S0034-71081999000300002

NIKOLAOU, A.D; LEKKKAS, T.D; GOLFINOPOULOS, S.K.; KOSTOPOULOU, M.N. (2002) Application of differentanalytical methods for determination of volatile chlorination by-products in drinking water. Talanta, v. 56, n. 4, p. 717-726. https://doi.org/10.1016/s0039-9140(01)00613-0

PASCHOALATO, C.F.P.R. (2005) Efeito da pré-oxidação, coagulação, filtração e pós-cloração na formação de subprodutos orgânicos halogenados em águas contendo substâncias húmicas. 291f. Tese (Doutorado em Engenharia Civil) - Escola de Engenharia de São Carlos, Universidade de São Paulo, São Carlos.

PAVÓN, J.L.P.; MARTINN, S.H.; PINTO, C.G.; CORDERO, B.M. (2008) Determination of trihalomethanes in water samples: a review. Analytical Chemistry Acta, v. 629, n. 1-2, p. 6-23. https://doi. org/10.1016/j.aca.2008.09.042

PLUMMER, J.D.; EDZWALD, J.K. (2001) Effect of ozone on algae as precursors for trihalomethane and haloacetic acid production. Environmental Science \& Technology, v. 35, n. 18, p. 3661-3668. https://doi.org/10.1021/es0106570

RIBANI, M.; BOTTOLI, C.B.G.; COLLINS, C.H.; JARDIM, I.C.S.F.; MELO, L.F.C. (2004) Validation for chromatographic and electrophoretic methods. Química Nova, v. 27, n. 5, p. 771-780.

RODRIGUES, F.M. (2014) Análise do risco da presença de trihalometanos em água para consumo humano, no distrito de Braga. 135f. Dissertação (Mestrado em Engenharia Civil) Universidade do Minho, Braga.

RODRIGUEZ, E.M.; ONSTAD, G.D.; KULL, T.P.J; METCALF, J.S.; ACERO, J.L.; VON GUNTEN, U. (2007) Oxidative elimination of cyanotoxins: comparison of ozone, chlorine, chlorine dioxide and permanganate. Water Research, v. 41, n. 15, p. 3381-3393. https://doi. org/10.1016/j.watres.2007.03.033

SANT'ANNA, C.L.; AZEVEDO, M.T.P.; WERNER, W.R.; DOGO, C.R.; RIOS, F.R.; CARVALHO, L.R. (2008) Review of toxic species of Cyanobacteria in Brazil. Algological Studies, v. 126, n. 1, p. 251-265. https://doi.org/10.1127/1864-1318/2008/0126-0251

SILVA, E.M.; GOMES, C.T.; RAMOS, C.P.; BRICIO, S.M. (2O13) Ocorrência de Cianobactérias no Reservatório de Mundaú, no Estado de Pernambuco, no período de janeiro de 2010 a novembro de 2011 Vigilância Sanitária em Debate, v. 1, n. 3, p. 35-42.

STEFFEN, M.M.; BELISLE, B.S.; WATSON, S.B., BOYER, G.L.; WILHELM, S.W. (2014) Status, causes and controls of cyanobacterial blooms in Lake Erie. Journal of Great Lakes Research, v. 40, n. 2, p. 215-225. https://doi.org/10.1016/j.jglr.2013.12.012

UNITED STATES ENVIRONMENTAL PROTECTION AGENCY (USEPA). (1995a) Method 524.2: Measurement of purgeable organic compounds in water by capillary column gas chromatography/ mass spectrometry. Cincinnati: USEPA.

UNITED STATES ENVIRONMENTAL PROTECTION AGENCY (USEPA). (1995b) Method 551.1: Determination of chlorination disinfection byproducts, chlorinated solvents, and halogenated pesticides/herbicides in drinking water by liquid-liquid extraction and gas chromatography with electron-capture detection. Cincinnati: USEPA.

UNITED STATES ENVIRONMENTAL PROTECTION AGENCY (USEPA) (1998) National Primary Drinking Water Regulations: Disinfectants and Disinfection Byproducts. Rules and Regulations. Cincinnati: USEPA.

UNITED STATES ENVIRONMENTAL PROTECTION AGENCY (USEPA). (2012) Edition of the Drinking Water Standards and Health Advisories. Washington, D.C.: USEPA.

YANG, X: GUO, W: SHEN, Q. (2O11) Formation of disinfection byproducts from chlor(am)ination of algal organic matter. Journal of Hazardous Materials, v. 197, p. 378-388. https://doi.org/10.1016/j.jhazmat.2011.09.098

ZAMYADI, A.; FAN, Y; DALY, R.I.; Prévost, M. (2013) Chlorination of Microcystis aeruginosa: Toxin release and oxidation, cellular chlorine demand and disinfection by-products formation Water Research, v. 47, n. 3, p. 1080-1090. https://doi.org/10.1016/j. watres.2012.11.031

ZANCHETTA, P.G.; PENA, A.; GONÇALVES, R.F. (2015) Desenvolvimento e validação de método para quantificação simultânea de ofloxacina, norfloxacina e ciprofloxacina em urina humana. Engenharia Sanitária e Ambiental, v. 20, n. 2, p. 307-314. http://dx.doi.org/10.1590/S1413-41522015020000114433 